\title{
EQUAÇÕES DE VOLUME DE POVOAMENTO PARA FRAGMENTOS FLORESTAIS NATURAIS DO MUNICÍPIO DE VIÇOSA, MINAS GERAIS ${ }^{1}$
}

\author{
Márcio Leles Romarco de Oliveira ${ }^{2}$, Carlos Pedro Boechat Soares ${ }^{3}$, Agostinho Lopes de Souza ${ }^{3}$ e Helio
}

Garcia Leite ${ }^{3}$

\begin{abstract}
RESUMO - Este trabalho teve por objetivos desenvolver equações de volume de povoamento em fragmentos florestais naturais no município de Viçosa, Minas Geras, utilizando-se a amostragem por pontos (Método de Bitterlich); caracterizar as famílias e as espécies arbóreas que ocorrem na região, fundamentadas em trabalhos científicos; e caracterizar os fragmentos florestais do município, quanto a tamanho, forma e parâmetros quantitativos. Para isso, foram amostrados 55 pontos, através da amostragem por pontos (Bitterlich), com um fator de área basal $K=1$. Após as análises, verificou-se que o município de Viçosa possui 480 espécies arbóreas, pertencentes a 189 gêneros e 62 famílias; e 289 fragmentos florestais recobrindo 22,77\% de área, equivalentes a 6.832,58 ha. O modelo, cujos volumes por hectare e as variáveis independentes área basal por hectare e altura do povoamento (altura total média e altura média dos fustes) estavam linearizados, foi selecionado como o melhor para estimar o volume total com casca por hectare e o volume de fuste com casca por hectare.
\end{abstract}

Palavras-chave: Equação de volume de povoamento, Bitterlich e florestas naturais.

\section{STAND VOLUME EQUATIONS FOR NATURAL FOREST FRAGMENTS IN THE MUNICIPALITY OF VICGOSA, MINAS GERAIS}

\begin{abstract}
Stand volume equation for natural forest fragments in the municipality of Viçosa, Minas Gerais, were developed with the point sampling (Bitterlich's method). Moreover, the tree families and species found in the region were identified, and forest fragments characterized according to size, shape, and quantitative parameters. For that, 55 points were sampled according to the point sampling method (Bitterlich), with a basal area factor $\mathrm{K}=1$. Results of the analyses evidenced 480 tree species in the municipality of Viçosa, distributed in 189 genera and 62 families. The municipality has 289 forest fragments which cover 22,77\% of its area $(6.832,58 \mathrm{ha})$. The model whose volumes per hectare, dependent basal area variables per hectare and height of the stand (mean total height and mean height of stems) had been linearized, was selected as the best model to estimate the total volume per hectare and bole volume outside bark per hectare.
\end{abstract}

Key words: Stand volume equation, Bitterlich, natural forests.

\footnotetext{
${ }^{1}$ Recebido em 07.11.2003 e aceito para publicação em 25.11.2004.

${ }^{2}$ Programa de Pós-Graduação em Ciência Florestal da UFV.

${ }^{3}$ Departamento de Engenharia Florestal da Universidade Federal de Viçosa - UFV, 36570-000 Viçosa-MG.
} 


\section{INTRODUÇÃO}

Para a realização do manejo florestal, visando à produção sustentável, é de fundamental importância conhecer as diversas características da floresta, as quais podem ser obtidas pelo inventário florestal, executado por meio da avaliação de parte da população, através de amostragem. A literatura é vasta quanto aos procedimentos de amostragem que podem ser utilizados na área florestal, contudo a escolha do procedimento depende das características da floresta, dos objetivos a serem alcançados, dos recursos disponíveis e da precisão requerida (HUSH et al., 1972).

Segundo Campos (1986), o método de Bitterlich e as equações ou tabelas de volume de povoamento são alternativas para a obtenção de características da floresta como número de árvores, área basal e volume por hectare, com precisão, com um menor custo e em menor tempo, quando comparados com os procedimentos convencionais de inventário, baseados na totalização de parcelas de área fixa.

Segundo Carron e Cromer (1956) com as tabelas de volume de povoamento podem-se obter estimativas do volume por unidade de área de forma rápida e simples. Cole (1971), por sua vez, destacou, além da rapidez e da simplicidade do uso das tabelas de volume de povoamento, a precisão, afirmando que esse método pode ser tão preciso quanto o inventário florestal convencional. Spurr (1952) ressaltou, ainda, que esse método pode ser usado para obtenção de estimativas adequadas e confiáveis para o manejo florestal, em comparação com o método que estima o volume do povoamento através da soma do volume das árvores individualmente.

Diante disso, o presente trabalho teve por objetivos desenvolver equações de volume de povoamento para fragmentos florestais naturais no município de Viçosa, Minas Geras, utilizando a amostragem por pontos (Método de Bitterlich); caracterizar as famílias e as espécies arbóreas que ocorrem na região, fundamentadas em trabalhos científicos; e caracterizar os fragmentos florestais do município, em termos de tamanho, forma e de parâmetros quantitativos.

\section{MATERIAL E MÉTODOS}

\subsection{Caracterização da área de estudo}

O presente trabalho foi desenvolvido na Zona da Mata mineira, município de Viçosa, Estado de Minas
Gerais, cuja sede administrativa está localizada a aproximadamente $42^{\circ} 53^{\prime} \mathrm{W}$ e $20^{\circ} 45^{\prime}$ 'S (PEREIRA, 1999).

A precipitação média anual do município é de aproximadamente $1.221,4 \mathrm{~mm}$, em sua maior parte, concentrada entre os meses de outubro e março, com a temperatura média anual oscilando entre 19 e $20^{\circ} \mathrm{C}$ (DEPARTAMENTO ..., 1992).

O clima, de acordo com a classificação de Köppen, é o tropical de altitude, com verões chuvosos e invernos frios e secos do tipo Cwb (GOLFARI, 1975).

A topografia local é acidentada, apresentando relevo forte ondulado e montanhoso, com vales estreitos e úmidos e altitude variando, aproximadamente, de 600 a $970 \mathrm{~m}$, estando o município a $649 \mathrm{~m}$ de altitude (CORRÊA, 1984).

Os solos do município de Viçosa apresentam a predominância de duas classes: o Latossolo VermelhoAmarelo álico predomina nos topos de morro e encostas, enquanto o Podzólico Vermelho-Amarelo câmbico predomina nos terraços (REZENDE, 1971). De acordo com a classificação da Embrapa (1999), o Latossolo Vermelho-Amarelo álico passou a ser classificado apenas como Latossolo e o Podzólico Vermelho-Amarelo câmbico, classificado como alissolo.

Pela classificação fitossociológica do RADAM BRASIL, o município está inserido na região fitossociológica classificada como Floresta Estacional Semidecidual (VELOSO et al., 1991). As formações florestais estão nos estágios primário e secundário, sendo os remanescentes florestais, em sua maioria, localizados em área de difícil acesso e de relevo acidentado (COELHOe SOUZA, 2002).

\subsection{Caracterização das espécies e dos fragmentos florestais no município de Viçosa}

A caracterização das espécies florestais que ocorrem no município de Viçosa foi elaborada com base em revisão bibliográfica, apresentada em Oliveira (2003).

Para caracterizar os fragmentos florestais do município de Viçosa foi utilizada uma base de dados digitais disponibilizada pelo Centro de Estudos e Desenvolvimento Florestal (CEDEF), do Instituto Estadual de Florestas (IEF/MG). Dessa base de dados, obtiveram-se o número total de fragmentos, o número de fragmentos por classe de tamanho, a área total coberta

R. Árvore, Viçosa-MG, v.29, n.2, p.213-225, 2005 
pelos fragmentos e a porcentagem de recobrimento pelas florestas no município, bem como o índice de circularidade, calculado através da seguinte expressão (CHATURVEDI, 1926):

$$
\mathrm{IC}=\frac{40000 \cdot \Pi \cdot \mathrm{A}}{\mathrm{P}^{2}}
$$

em que:

$\mathrm{IC}=$ índice de circularidade; $\mathrm{A}=$ área do fragmento, em ha; e $\mathrm{P}=$ perímetro do fragmento, em metros.

\subsection{Amostragem e coleta dos dados}

Na coleta dos dados de campo, empregou-se a amostragem por ponto horizontal, através do método de Bitterlich. Foram amostrados 55 pontos, dos quais 41 foram lançados em fragmentos que possuíam descrição sobre a florística e fitossocilogia e utilizados no ajuste das equações e 14 em fragmentos selecionados aleatoriamente no município, para a validação destas.

Para a obtenção das informações em cada ponto de amostragem, foi utilizado um relascópio do tipo Standard, empregando-se um fator de área basal igual a $1(K=1)$. O nível de inclusão foi de $5 \mathrm{~cm}$ de $D A P$ (diâmetro a 1,30 $\mathrm{m}$ do solo). Em cada ponto de amostragem e para cada árvore qualificada, obtiveram-se as estimativas do $C A P$ (circunferência a $1,30 \mathrm{~m}$ do solo), da altura total e da altura do fuste.

As circunferências foram medidas com fita diamétrica $\mathrm{e}$, às vezes, trena. As alturas totais e dos fustes das árvores foram estimadas, baseando-se em vara de náilon de $6 \mathrm{~m}$ de comprimento. A altura de fuste compreendeu a distância da superfície do solo até a primeira bifurcação ou até a inserção dos primeiros galhos.

\subsection{Estimativas dos parâmetros populacionais}

As estimativas dos parâmetros populacionais, área basal por hectare $(B)$, número de árvores por hectare $(N)$, volume total com casca por hectare $(V t)$, volume de fuste com casca por hectare $(V f)$, altura total média $(\bar{H} t)$ e altura média dos fustes das árvores $(\bar{H} f)$ em cada ponto de amostragem, foram calculadas de acordo com o Quadro 1.

Segundo o Cetec (1995), no estágio sucessional em que se encontra a grande maioria dos fragmentos florestais da região de Viçosa (secundário), pode-se calcular o volume total com casca e o volume do fuste com casca de árvores individuais pelo emprego das seguintes equações:

$$
\begin{aligned}
& V t=0,000074230 * D A P^{1,707348 * H} t^{1,16873} \quad \mathrm{R}^{2}=97,3 \\
& V t=0,000038857 * D A P^{1,70764 *} H f^{1,32032} \quad \mathrm{R}^{2}=98,9
\end{aligned}
$$$$
\text { em que: }
$$

$V t=$ volume total com casca, $\mathrm{em} \mathrm{m}^{3} ; D A P=$ diâmetro com casca, medido a $1,30 \mathrm{~m}$ do solo, em $\mathrm{cm} ; H t=$ altura total, em $\mathrm{m} ; V f=$ volume do fuste com casca, em $\mathrm{m}^{3}$; e $H f=$ altura do fuste, em metros.

\subsection{Equação de volume de povoamento}

Preliminarmente, foram realizadas algumas análises exploratórias para detectar a presença de outliers, bem como se estimou o coeficiente de correlação linear entre as variáveis, com a finalidade de observar o grau de associação entre as variáveis dependentes e independentes. Além disso, foram elaborados gráficos de dispersão, visando identificar padrões de distribuição entre as variáveis.

Quadro 1 - Estimadores dos parâmetros populacionais para o método de Bitterlich

Table 1 - Estimators of the populational parameters, for Bitterlich's Method

\begin{tabular}{ll}
\hline \multicolumn{1}{c}{ Variável } & \multicolumn{1}{c}{ Fórmula } \\
\hline Área basal por hectare & $B=n^{*} K$ \\
Número de árvores por hectare & $N=\sum_{i=1}^{n} \frac{K}{A s_{i}}=\sum_{i=1}^{n} N_{i}$ \\
Volume total por hectare & $V / h a=\sum_{i=1}^{n} V t_{i} * N_{i}$ \\
Volume de fuste por hectare & $V f=\sum_{i=1}^{n} V f_{i} * N_{i}$ \\
Altura total média & $\bar{H} t=\frac{\sum_{i=1}^{n} H t_{i}}{n}$ \\
Altura média de fuste & $\bar{H} f=\frac{\sum_{i=1}^{n} H f_{i}}{n}$ \\
\hline
\end{tabular}

em que: $n=$ número de árvores qualificadas nos pontos de amostragem; $K=$ fator de área basal, $\mathrm{em} \mathrm{m}^{2} / \mathrm{ha} ; N_{i}=$ número de árvores por hectare que a i-ésima árvore qualificada representa; $A s_{i}=$ área seccional da i-ésima árvore qualificada, $\mathrm{em}^{2}$; $V t_{i}$ e $V f_{i}=$ volume total com casca e volume do fuste com casca, respectivamente, da i-ésima árvore qualificada, $\mathrm{em}^{3}$; e $H t_{i}$ e $H f_{i}=$ altura total e altura do fuste, respectivamente, da i-ésima árvore qualificada, em $\mathrm{m}$.

R. Árvore, Viçosa-MG, v.29, n.2, p.213-225, 2005 
Em seguida foram testados modelos lineares e nãolineares encontrados na literatura (Quadro 2), os quais foram ajustados utilizando o software STATISTICA versão 5.0 .

A escolha da melhor equação foi feita com base nos seguintes critérios: a) menor coeficiente de variação $(C V)$; b) significância das variáveis do modelo; c) maior coeficiente de determinação $\left(R^{2}\right)$; d) coerência dos sinais associados aos parâmetros; e e) análise gráfica dos resíduos porcentuais $(R \%)$, calculados pela seguinte expressão:

$$
R(\%)=\frac{\hat{V}-V}{V} .100
$$

em que:

$\widehat{V}=$ volume estimado, em $\mathrm{m}^{3} /$ ha; e $V=$ volume observado, em $\mathrm{m}^{3} / \mathrm{ha}$.

Como alguns modelos lineares possuem variáveis dependentes linearizadas e alguns modelos são nãolineares, houve a necessidade de calcular o coeficiente de determinação $\left(R^{2}\right)$ e o coeficiente de variação $(C V)$, através das seguintes expressões, para possibilitar a comparação das equações.

$$
\begin{aligned}
& R^{2}=\left(r_{v \hat{v}}\right)^{2} \\
& r_{\hat{v} \hat{v}}=\frac{\sum v \hat{v}-\frac{\left(\sum v\right)\left(\sum \hat{v}\right)}{n}}{\sqrt{\left(\sum v^{2}-\frac{\left(\sum v\right)^{2}}{n}\right)\left(\sum \hat{v}^{2}-\frac{\left(\sum \hat{v}\right)^{2}}{n}\right)}}
\end{aligned}
$$

$$
C V(\%)=\frac{\sqrt{\frac{\sum(v-\hat{v})^{2}}{n-p-1}}}{\bar{v}} .100
$$

em que:

$r=$ coeficiente de correlação linear $\hat{v}=$ volume estimado, $\mathrm{em} \mathrm{m}^{3} / \mathrm{ha} ; v=$ volume observado, $\mathrm{em} \mathrm{m}^{3} / \mathrm{ha}$; $n=$ número de observações; $p=$ número de variáveis independentes; e $\bar{v}=$ média artimética do volume observado, em $\mathrm{m}^{3} / \mathrm{ha}$.

\subsection{Validação das equações ajustadas}

Com as melhores equações para estimar o volume total com casca e o volume do fuste com casca dos fragmentos, fez-se a validação das equações, utilizando dados de 14 pontos de amostragem independentes (não entraram no ajuste).

A validação se deu pela análise gráfica dos resíduos porcentuais, conforme definido na equação 4, pela verificação das amplitudes dos resíduos e pela tendência da distribuição.

\section{RESULTADOS E DISCUSSÃO}

\subsection{Caracterização das espécies florestais no município de Viçosa}

Com base na literatura consultada, foram contabilizadas 480 espécies, pertencentes a 189 gêneros e 62 famílias. Das espécies listadas, 382 (79,58\%) foram identificadas em nível de espécie, 82 (17,09\%) em nível

\begin{tabular}{|c|c|c|}
\hline № & Modelo & Autor \\
\hline 1 & $V=\beta_{o}+\beta_{1} \cdot B \cdot H+\varepsilon$ & Spurr (1952) \\
\hline 2 & $V=\beta_{0}+\beta_{1} \cdot \operatorname{Ln} B \cdot H+\varepsilon$ & Figueredo Filho (1983) \\
\hline 3 & $V=\beta_{0}+\beta_{1} \cdot \operatorname{Ln} B+\varepsilon$ & Figueredo Filho (1983) \\
\hline 4 & $\operatorname{Ln}(V)=\beta_{0}+\beta_{1} \cdot \operatorname{Ln} B \cdot H+\varepsilon$ & Figueredo Filho (1983) \\
\hline 5 & $\operatorname{Ln}(V)=\beta_{0}+\beta_{1} \cdot \operatorname{Ln} B+\varepsilon$ & Figueredo Filho (1983) \\
\hline 6 & $\operatorname{Ln}(V)=\beta_{0}+\beta_{1} \cdot \operatorname{Ln} B+\beta_{2} \cdot \operatorname{Ln} H+\varepsilon$ & Ung e Ouellet (1991)* \\
\hline 7 & $V=\beta_{o}+\beta_{1} \cdot B+\beta_{2} \cdot H+\beta_{3} \cdot B \cdot H+\varepsilon$ & Spurr (1952) \\
\hline 8 & $V=e^{\beta_{0} \cdot B^{\beta_{1}} \cdot H^{\beta_{2}}}+\varepsilon$ & Ung e Quellet (1991) \\
\hline
\end{tabular}
de gênero e $16(3,33 \%)$ em nível de família.

Quadro 2 - Modelos testados para estimar o volume de povoamento Table 2 - Studied models to estimate the stand volume

*linearizado.

Em que: $V=$ volume total com casca por hectare ou volume do fuste com casca por hectare, em $\mathrm{m}^{3} / \mathrm{ha} ; B=$ área basal por hectare, em $\mathrm{m}^{2} / \mathrm{ha} ; H=$ altura total média das árvores ou altura média do fuste, em $\mathrm{m} ; \mathrm{Ln}=1 \log$ aritmo neperiano; $\beta_{0} \ldots$ $\beta_{3}=$ parâmetros do modelo; e $\varepsilon=$ erro aleatório.

R. Árvore, Viçosa-MG, v.29, n.2, p.213-225, 2005 
As seis principais famílias em número de espécies são: Leguminosae, com 76 espécies $(15,83 \%), 21$ pertencentes à subfamília Caesalpinioideae, 25 à Mimosoideae e 30 à Papilionideae; Myrtaceae, com 41 espécies (8,54\%); Lauraceae, com 40 espécies $(8,33 \%)$; Rubiaceae, com 27 espécies (5,63\%); e Euphorbiaceae, com $25(5,21 \%)$. Essas famílias contribuíram com 43,54\% do total de espécies.

As famílias Labiateae, Lacistemataceae, Lamiaceae, Olacaceae, Opiliaceae, Piperaceae, Protecaceae e Ulmaceae apresentaram apenas uma espécie. Os gêneros mais abundantes, ou seja, aqueles com mais espécies, foram: Ocotea (Lauraceae), com 18 espécies e Inga (Leguminosae mimosoideae); e Machaerium (Leguminosae papilionideae), com 12 espécies.

De acordo com a "Lista Oficial de Espécies Brasileiras Ameaçadas de Extinção” (IBAMA,2002), foram encontradas três espécies ameaçadas, Astronium flaxinifolium (Anacardiaceae), Dalbergia nigra (Leguminoseae papilionideae) e Melanoxylom brauna (Leguminosae caesalpinioideae).

\subsection{Caracterização dos fragmentos florestais}

Os fragmentos florestais foram divididos em classes de tamanho com amplitude de 10 ha, totalizando 18 classes. O município de Viçosa apresenta, segundo dados fornecidos pelo IEF-MG, 289 fragmentos florestais com área maior que 0,5 ha. Desses, 142 (49,13\%) possuem área de até 10 ha (centro de classe 5 ha). Sete classes de tamanho possuem apenas um fragmento. Apesar de a primeira classe apresentar o maior número de fragmentos, estes contribuíram com 9,84\% da área total da cobertura florestal. A maior contribuição em termos de área de cobertura florestal se deve aos fragmentos com área entre 10 e 20 ha (centro de classe 15 ha), com $11,40 \%$ da área total. A maior classe de tamanho possui apenas um fragmento, representado $6,25 \%$ da área total (Quadro 3).

Fragmentos com até 30 ha são considerados relativamente pequenos, podendo apresentar baixa capacidade de sustentabilidade (SAUNDERS et al., 1991). O município de Viçosa apresenta 229 fragmentos menores que 30 ha, isto é, 79,24\% dos fragmentos.

Quadro 3-Distribuição dos fragmentos florestais do município de Viçosa, MG, quanto a classe de tamanho, índice de circularidade médio (IC) e contribuição porcentual

Table 3 -Distribution of the forest fragments of the municipality of Viçosa-MG in terms of size class; index of average circularity (IC) and percentile of contribution

\begin{tabular}{|c|c|c|c|c|c|c|c|c|}
\hline $\begin{array}{c}\text { Classe } \\
\text { (ha) }\end{array}$ & $\begin{array}{l}\text { Centro de } \\
\text { Classe (ha) }\end{array}$ & $\begin{array}{c}\mathrm{N}^{\circ} \\
\text { Fragmentos }\end{array}$ & $\begin{array}{c}\% \text { de } \\
\text { Fragmentos }\end{array}$ & $\begin{array}{c}\text { IC } \\
\text { Médio }\end{array}$ & $\begin{array}{c}\Sigma \text { da área } \\
\text { (ha) }\end{array}$ & $\begin{array}{l}\% \text { de } \\
\text { Área }\end{array}$ & $\begin{array}{l}\text { \% de área } \\
\text { Acumulada }\end{array}$ & $\begin{array}{c}\text { Área Média } \\
\text { (ha) }\end{array}$ \\
\hline $0-10$ & 5 & 142 & 49,13 & 0,68 & 672,32 & 9,84 & 9,84 & 4,73 \\
\hline $10-20$ & 15 & 57 & 19,72 & 0,55 & 779,23 & 11,40 & 21,24 & 13,67 \\
\hline $20-30$ & 25 & 30 & 10,38 & 0,44 & 726,70 & 10,64 & 31,88 & 24,22 \\
\hline $30-40$ & 35 & 18 & 6,23 & 0,38 & 623,06 & 9,12 & 41,00 & 34,61 \\
\hline $40-50$ & 45 & 12 & 4,15 & 0,32 & 534,57 & 7,82 & 48,82 & 44,55 \\
\hline $50-60$ & 55 & 5 & 1,73 & 0,26 & 282,52 & 4,13 & 52,96 & 56,50 \\
\hline $60-70$ & 65 & 4 & 1,38 & 0,23 & 266,94 & 3,91 & 56,86 & 66,73 \\
\hline $70-80$ & 75 & 6 & 2,08 & 0,24 & 448,04 & 6,56 & 63,42 & 74,67 \\
\hline $80-90$ & 85 & 4 & 1,38 & 0,25 & 337,04 & 4,93 & 68,36 & 84,26 \\
\hline $90-100$ & 95 & 1 & 0,35 & 0,14 & 93,42 & 1,37 & 69,72 & 93,42 \\
\hline $100-110$ & 105 & 2 & 0,69 & 0,35 & 209,22 & 3,06 & 72,78 & 104,61 \\
\hline $110-120$ & 115 & 2 & 0,69 & 0,24 & 235,24 & 3,44 & 76,23 & 117,62 \\
\hline $120-130$ & 125 & 1 & 0,35 & 0,15 & 126,79 & 1,86 & 78,08 & 126,79 \\
\hline $140-150$ & 145 & 1 & 0,35 & 0,24 & 149,48 & 2,19 & 80,27 & 149,48 \\
\hline $200-210$ & 205 & 1 & 0,35 & 0,10 & 204,40 & 2,99 & 83,26 & 204,40 \\
\hline $310-320$ & 315 & 1 & 0,35 & 0,07 & 310,55 & 4,55 & 87,81 & 310,55 \\
\hline $400-410$ & 405 & 1 & 0,35 & 0,12 & 405,77 & 5,94 & 93,75 & 405,77 \\
\hline $420-430$ & 425 & 1 & 0,35 & 0,12 & 427,28 & 6,25 & 100,00 & 427,28 \\
\hline Total & & 289 & 100,00 & & 6832,58 & 100,00 & & \\
\hline
\end{tabular}

R. Árvore, Viçosa-MG, v.29, n.2, p.213-225, 2005 
Apresenta também um porcentual elevado de fragmentos menores que 10 ha $(49,13 \%)$. Segundo Pereira (1999), fragmentos com área de até 10 ha têm $90 \%$ de sua área afetada pelo efeito de borda. Além disso, Viana et al. (1992) destacaram que pequenos fragmentos florestais apresentam problemas quanto ao tamanho das populações, por conterem poucos indivíduos, podendo acontecer, dentro de certo período de tempo, o declínio das populações, resultando na perda de biodiversidade.

Segundo dados preliminares do último censo realizado em 2000 pelo Instituto Brasileiro de Geografia e Estatística (IBGE), o município de Viçosa tem área igual a $300 \mathrm{~km}^{2}$. De acordo com o Quadro 3, a área total dos fragmentos florestais é de $6.832,58$ ha, equivalendo a $22,77 \%$ da área do município. Esse valor está próximo ao indicado por Coelho e Souza (2002), que destacaram que a microrregião de Viçosa possui, aproximadamente, $23,01 \%$ de cobertura florestal nativa.

Ainda de acordo com o Quadro 3, fragmentos de tamanho menor apresentaram, em média, maiores valores para os índices de circularidade (IC). Fragmentos com valores de índice de circularidade mais próximos de 1 são mais arredondados e apresentam menor relação borda/interior, estando sujeitos a um menor efeito de borda (ALMEIDA JUNIOR, 1999). O maior fragmento de Viçosa, que pertence à classe de tamanho com centro de 425 ha, apresenta índice de circularidade em torno de 0,1 , ou seja, tem alta relação borda/interior.

Viana e Pinheiro (1998) estudaram a forma de fragmentos florestais na região de Piracicaba, utilizando o parâmetro fator de forma que é a relação entre a área do fragmento e seu perímetro. Os fragmentos foram classificados da seguinte forma: fragmentos com fator de forma superior a 0,8 são considerados arredondados; entre 0,6 e 0,8 são alongados e inferior a 0,6 , muito alongados. Adotando essa classificação no presente trabalho e analisando a Figura 1, podem-se considerar arredondados apenas alguns fragmentos com até 10 ha. Alguns fragmentos até 20 ha podem ser considerados alongados, e todos os fragmentos acima de 20 ha são classificados como muito alongados.

\subsection{Caracterização de parâmetros quantitativos}

Analisando o Quadro 4, verifica-se, para as variáveis $D A P$, altura total e altura de fuste, uma amplitude grande entre os valores amostrados. Considerando os valores médios para a altura total e para a altura de fuste $(11,80$

R. Árvore, Viçosa-MG, v.29, n.2, p.213-225, 2005 m e 7,53 m, respectivamente) das árvores amostradas, verificou-se que os fragmentos são relativamente baixos, refletindo em uma primeira análise o estágio de sucessão e o estado de conservação dos fragmentos florestais no município de Viçosa.

\subsubsection{Distribuição diamétrica}

De acordo com a Figura 2, a amostragem por pontos com fator de área basal igual a $1(\mathrm{~K}=1)$ caracterizou a distribuição diamétrica como tendo uma tendência a J-invertido, que é a distribuição característica de florestas ineqüiâneas (DAVIS e JONHSON, 1986). A distribuição diamétrica só não foi um J-invertido perfeito, devido ao fato de a primeira classe $(7,5 \mathrm{~cm})$ apresentar menor número de indivíduos que a classe seguinte $(12,5 \mathrm{~cm})$. Isso pode indicar que alguns locais sofreram alterações na sua estrutura, pelo corte seletivo de árvores de menor porte, para a produção de energia.

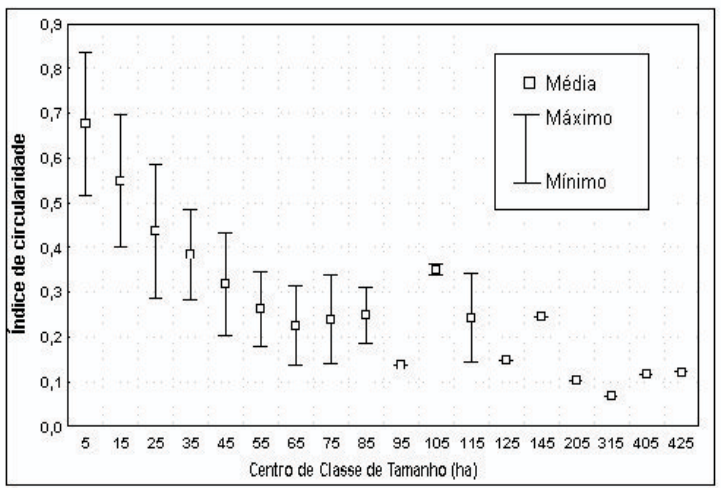

Figura 1 - Dispersão dos valores de índice de circularidade por classe de tamanho.

Figure 1 - Value dispersion of the circularity index according to size class.

Quadro 4 - Valores mínimos, médios e máximos observados de variáveis, $D A P$, altura total $(H t)$ e de fuste $(H f)$

Table 4-Minimum, medium, and maximum values observed for the variables, DBH, total and bole height

\begin{tabular}{lccc}
\hline Variáveis & Mínimo & Máximo & Médio \\
\hline$D A P(\mathrm{~cm})$ & 5,09 & 98,04 & 19,73 \\
$H t(\mathrm{~m})$ & 3,80 & 32,00 & 11,80 \\
$H f(\mathrm{~m})$ & 1,50 & 18,00 & 7,53 \\
\hline
\end{tabular}

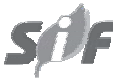




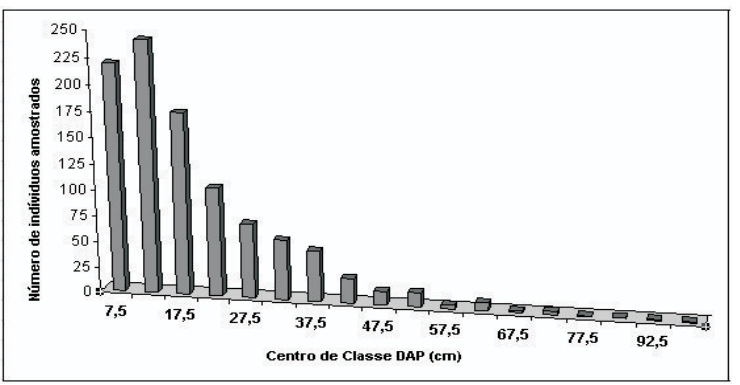

Figura 2 - Distribuição diamétrica considerando todos os indivíduos amostrados.

Figure 2 -Diametric distribution including all sampled individuals.

\subsection{Caracterização de parâmetros populacionais}

Os valores mínimos, médios e máximos, bem como o desvio-padrão das variáveis altura total média $(\bar{H} t)$, altura média do fuste $(\bar{H} f)$, área basal por hectare $(B)$, número de árvores por hectare $(N)$, volume total com casca por hectare $(V t)$ e volume de fuste com casca por hectare $(V f)$, para os 41 pontos destinados ao ajuste dos modelos, encontram-se no Quadro 5.

Pela dispersão dos valores apresentados no Quadro 5 , verificou-se que a amostragem por pontos horizontais, através do método de Bitterlich, mais uma vez evidenciou os baixos valores das variáveis altura total média das árvores $(\bar{H} t)$ e altura média dos fustes $(\bar{H} f)$, o qual reflete o estado de conservação e desenvolvimento dos fragmentos. Além disso, verificou-se que, em média, o volume do fuste com casca representa cerca de $44 \%$ do volume total com casca por hectare.

Quadro 5-Amplitude de valores de parâmetros populacionais de fragmentos florestais amostrados no município de Viçosa

Table 5 - Populational parameters values range for forest fragments sampled in the municipality of Viçosa

\begin{tabular}{lcccc}
\hline Variáveis & \multicolumn{4}{c}{ Valores } \\
\cline { 2 - 5 } & Mínimo & Médio & Máximo & $\begin{array}{c}\text { Desvio- } \\
\text { Padrão }\end{array}$ \\
\hline $\bar{H} t(\mathrm{~m})$ & 7,33 & 11,97 & 18,27 & 2,56 \\
$\bar{H} f(\mathrm{~m})$ & 4,72 & 7,68 & 11,50 & 1,55 \\
$B\left(\mathrm{~m}^{2} / \mathrm{ha}\right)$ & 9,00 & 18,88 & 31,00 & 5,07 \\
$N$ & 648,14 & 1460,97 & 3403,97 & 582,14 \\
$V t\left(\mathrm{~m}^{3} / \mathrm{ha}\right)$ & 42,51 & 141,42 & 291,61 & 57,44 \\
$V f\left(\mathrm{~m}^{3} / \mathrm{ha}\right)$ & 16,99 & 62,18 & 120,11 & 25,62 \\
\hline
\end{tabular}

Os gráficos de dispersões na Figura 3 indicam uma correlação positiva entre a área basal por hectare, a altura média total das árvores e a altura média dos fustes com as variáveis volume total com casca por hectare e volume do fuste com casca por hectare. Podese observar, na Figura 3, que locais com mesma área basal possuem volumes diferentes, o mesmo ocorrendo com as variáveis altura total média e altura média dos fustes. Essa dispersão dos volumes para um mesmo valor de área basal e altura reflete a variabilidade natural dos fragmentos, fato desejável para o ajuste dos modelos volumétricos.

Na matriz de correlação linear (Quadro 6), verificouse que a área basal por hectare $(B)$ possui alta correlação com o volume total com casca por hectare $(V t)$ e o volume de fuste com casca por hectare $(V f)$. A altura total média $(\bar{H} t)$, por sua vez, é mais correlacionada com o volume total com casca por hectare $(r=0,85)$, enquanto a altura média do fuste $(\bar{H} f)$ é mais correlacionada com o volume do fuste com casca por hectare $(r=0,87)$. No entanto, dada a facilidade quanto à obtenção da altura do fuste em florestas nativas, esta pode ser usada na obtenção do volume total por hectare, embora apresente menor correlação com essa variável do povoamento; em comparação com a altura total, como apresenta correlação acima de $80 \%$, pode propiciar um bom poder de explicação, dependendo do modelo ajustado.

\subsection{Ajuste dos modelos}

Os modelos apresentados no Quadro 2, no item 2.5., foram ajustados utilizando-se, inicialmente, como variável dependente o volume total com casca por hectare em função da área basal e da altura total média do povoamento, quando pertinente. Analisando o Quadro 7, em que estão os coeficientes estimados, bem como as respectivas medidas de precisão, verificou-se que as equações resultantes estimam o volume total com casca por hectare e apresentam coeficientes de variação entre 5,32 e $20,30 \%$ e coeficientes de determinação $\left(R^{2}\right)$ entre 75,01 e $99,15 \%$. As equações que forneceram os piores ajustes foram as que tinham como variável independente apenas a área basal por hectare e suas transformações (equações 3 e 5).

As equações que mais se destacaram foram as de número 1, 6, 7 e 8 , por exibirem os menores valores de $C V(\%)$ e maiores valores de $R^{2}(\%)$. Porém, o modelo 7 apresenta problema de multicolinearidade devido à 
não-significância dos coeficientes da equação, embora apresente alto coeficiente de determinação. As equações 1 e 8 exibem resíduos (Figura 4) semelhantes, tendendo a uma pequena superestimação em locais com baixo estoque volumétrico. A equação 6, por sua vez, não apresenta essa tendência quanto à distribuição dos resíduos e possui o maior coeficiente de determinação

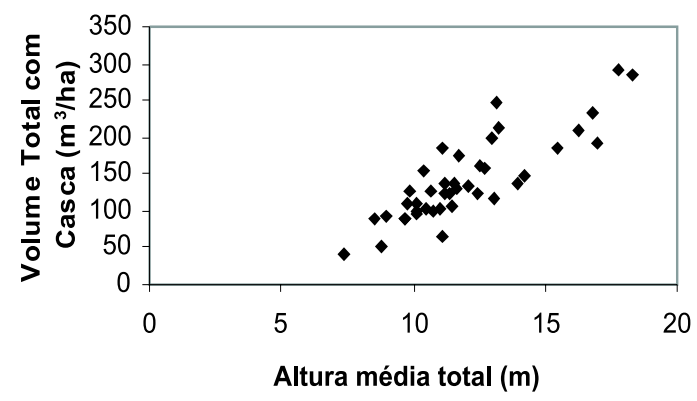

(A)

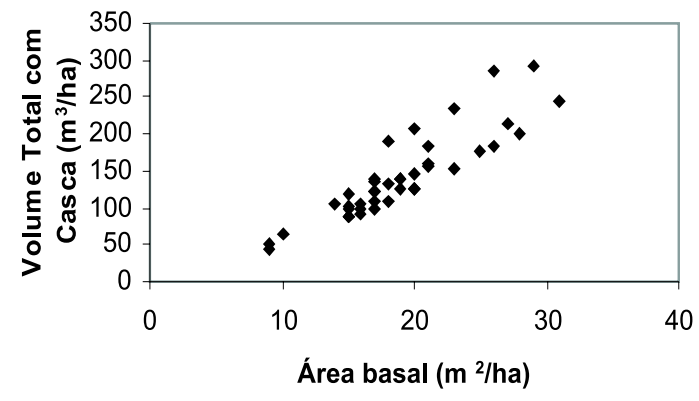

(C)
$\left(R^{2}\right)$. Assim, esse foi selecionado como o melhor modelo.

Substituindo a altura total média das árvores $(\mathrm{Ht})$ nos modelos 1, 2, 4, 6, 7 e 8 pela altura de média do fuste $(H f)$, obtiveram-se novas estimativas para os respectivos parâmetros dos modelos, bem como novas medidas de precisão, as quais estão apresentadas no Quadro 8.

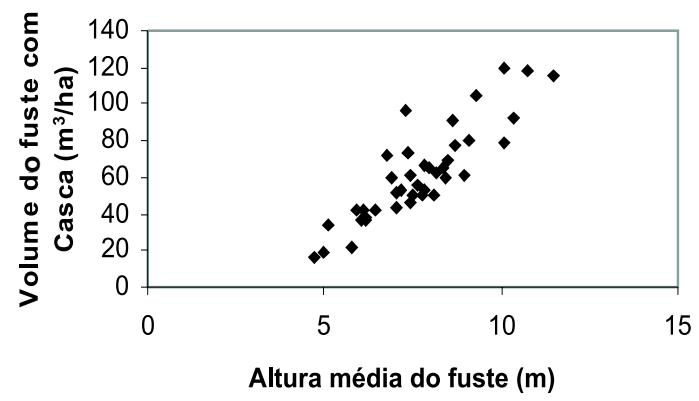

(B)

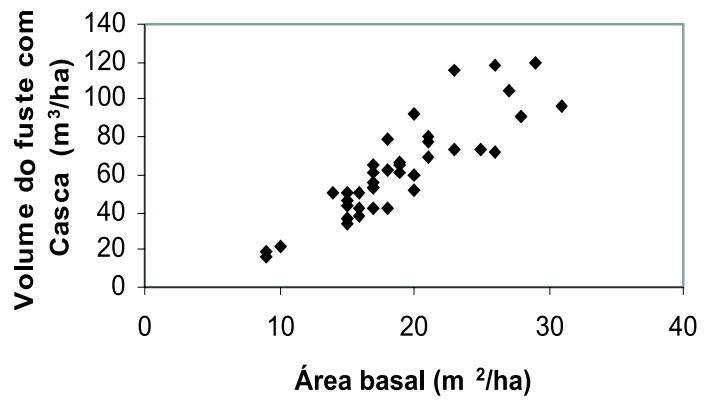

(D)

Figura 3 - Dispersão do volume total com casca por hectare em relação à altura total média das árvores (A) e à área basal por hectare dos pontos $(\mathrm{C})$ e dos volumes do fuste com casca por hectare em relação à altura média dos fustes (B) e à área basal por hectare dos pontos (D).

Figure 3 -Dispersion of the total volume with bark per hectare in relation to the total average height of trees $(A)$ and the basal area per hectare of the sample points $(C)$; and of the bole volumes with bark per hectare in relation to the average bole height $(B)$ and the basal area per hectare of the sample points $(D)$.

Quadro 6 - Matriz de correlação linear simples Table 6 - Simple linear correlation matrix

\begin{tabular}{lccccc}
\hline & $\boldsymbol{V} \boldsymbol{t}$ & $\boldsymbol{V} \boldsymbol{f}$ & $\bar{H} t$ & $\bar{H} f$ & $\boldsymbol{B}$ \\
\hline$V t$ & 1,00 & & & & \\
$V f$ & 0,97 & 1,00 & & & \\
$\bar{H} t$ & 0,85 & 0,82 & 1,00 & & \\
$\bar{H} f$ & 0,81 & 0,87 & 0,92 & 1,00 & \\
$B$ & 0,90 & 0,88 & 0,58 & 0,58 & 1,00 \\
\hline
\end{tabular}

Com exceção da equação 4 , as demais tiveram menor coeficiente de determinação $\left(R^{2}\right)$ em relação ao ajuste, utilizando a altura total. Tal fato se deve à menor correlação da altura média dos fustes com o volume total com casca por hectare, conforme mostrado no Quadro 6 (matriz de correlação). No entanto, os ajustes foram satisfatórios, uma vez que alguns modelos apresentaram $R^{2}$ maior que $95 \%$ (equações $1,6,7$ e 8 ).

R. Árvore, Viçosa-MG, v.29, n.2, p.213-225, 2005 
Equações de volume de povoamento para fragmentos...

Quadro 7 - Equações ajustadas do volume total $(V t)$ por hectare em função da área basal por hectare $(B)$ e da altura total média das árvores $(H t)$ quando pertinente, com os respectivos coeficientes de determinação $\left(R^{2}\right)$ e coeficientes de variação $(C V)$

Table 7 -Adjusted equations for total volume $(V t)$ per hectare in relation to the basal area per hectare $(B)$ and total average height of trees $(\mathrm{Ht})$ when pertinent, with their respective determination coefficients $\left(R^{2}\right)$ and variation coefficients $(\mathrm{CV})$

\begin{tabular}{|c|c|c|c|}
\hline № & Equações & $R^{2}(\%)$ & $C V(\%)$ \\
\hline 1 & $V t=8,924071 *+0,568072 * B . H t$ & 98,29 & 5,32 \\
\hline 2 & $V t=-54,355931^{*}+5,572753 * . \mathrm{LnB} . \mathrm{Ht}$ & 90,71 & 12,38 \\
\hline 3 & $V t=-376,630863 *+178,551789 * . \operatorname{LnB}$ & 75,01 & 20,30 \\
\hline 4 & $\operatorname{LnVt}=3,493448^{*}+0,039218^{*} . \mathrm{LnB} \cdot \mathrm{Ht}$ & 93,18 & 16,61 \\
\hline 5 & $\operatorname{LnVt}=0,869487^{*}+1,379240^{*} \cdot \operatorname{Ln} B$ & 89,48 & 18,40 \\
\hline 6 & $\operatorname{LnVt}=-0,156076^{*}+0,979917^{*} \cdot \operatorname{LnB}+0,887417^{*} \cdot \operatorname{LnH} t$ & 99,15 & 5,41 \\
\hline 7 & $V t=-12,596690^{n \cdot s}+1,227382^{n \cdot s} \cdot B+1,654361^{n . s} \cdot H t+0,476107^{*} \cdot B \cdot H t$ & 98,26 & 5,36 \\
\hline 8 & $V t=1,817886^{*} \cdot B^{0,186129 *} \cdot H t^{0,180576 *}$ & 99,08 & 5,64 \\
\hline
\end{tabular}

* Valores dos coeficientes significativos a $5 \%$, pelo teste t.

n.s Valores dos coeficientes não-significativos a 5\%, pelo teste t.
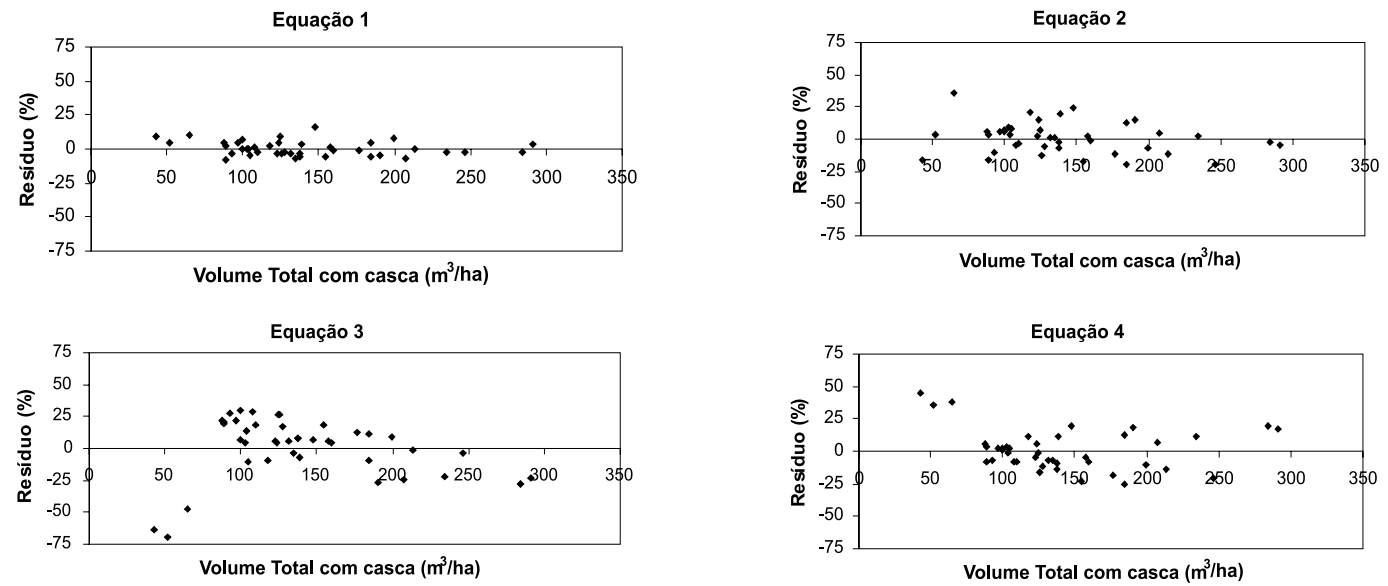

Equação 5

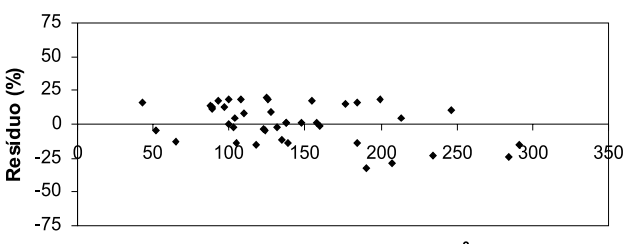

Equação 6

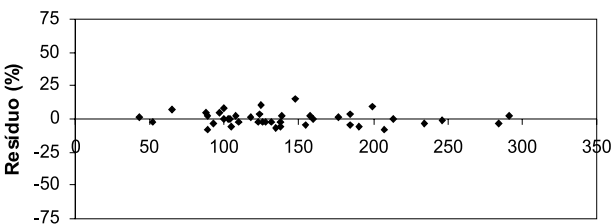

Volume Total com casca $\left(\mathrm{m}^{3} / \mathrm{ha}\right)$

Equação 7
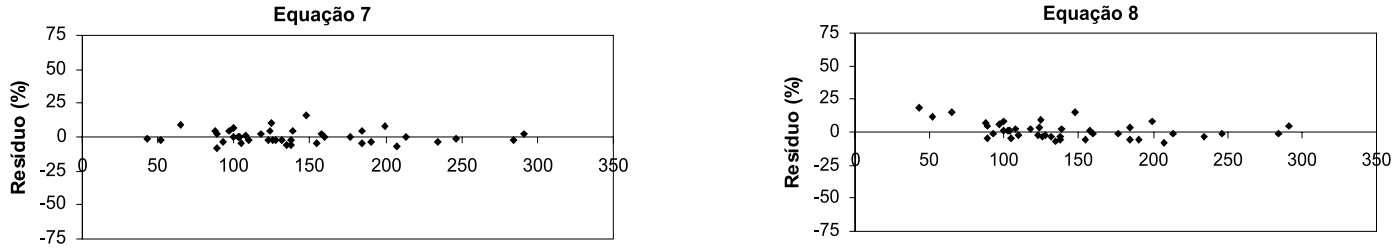

Figura 4 - Distribuição dos resíduos porcentuais para os modelos avaliados.

Figure 4 -Distribution of the percentile residues for evaluated models. 
Quadro 8 - Equações ajustadas de volume total por hectare $(V t)$ em função da área basal por hectare $(B)$ e da altura média dos fustes $(H f)$, com os respectivos coeficientes de determinação $\left(R^{2}\right)$ e coeficientes de variação $(C V)$

Table 8 -Adjusted equations for total volume $(V t)$ per hectare in relation to basal area per hectare $(B)$ and total average height of boles $(H f)$ when pertinent, with their respective determination coefficients $\left(R^{2}\right)$ and variation coefficients $(\mathrm{CV})$

\begin{tabular}{|c|c|c|c|}
\hline № & Equações & $R^{2}(\%)$ & $C V(\%)$ \\
\hline 1 & $V t=2,694030^{n . s}+0,928317^{*} \cdot B \cdot H f$ & 95,23 & 8,87 \\
\hline 2 & $V t=-57,784066^{*}+8,835160 * . \operatorname{LnB} . \mathrm{Hf}$ & 84,12 & 16,18 \\
\hline 4 & $L n V t=3,407264 *+0,064930 * . \operatorname{LnB} . H f$ & 91,21 & 17,47 \\
\hline 6 & $L n V t=0,326587 *+0,969486^{*} \cdot \operatorname{LnB}+0,857922 * . \operatorname{LnHf}$ & 97,59 & 9,43 \\
\hline 7 & $V t=24,639079^{n . s}-0,548850^{n . s} . B-4,041413^{n . s} \cdot H f+1,058513^{*} . B . H f$ & 95,15 & 8,94 \\
\hline 8 & $V t=1,835417^{*} \cdot B^{0,208280^{*}} \cdot H f^{0,182406^{*}}$ & 97,84 & 8,62 \\
\hline
\end{tabular}

* Valores dos coeficientes significativos a $5 \%$, pelo teste $\mathrm{t}$;

n.s Valores dos coeficientes não-significativos a 5\%, pelo teste t.

Mais uma vez, verificou-se o problema da multicolinearidade para a equação 7 pela não-significância de seus parâmetros.

Pela análise gráfica dos resíduos (Figura 5) e pelas medidas de precisão (Quadro 8), verificou-se que a equação 8 foi a melhor. Contudo, o modelo 6 , que é uma forma linear do modelo 8 , também apresentou alto ajuste

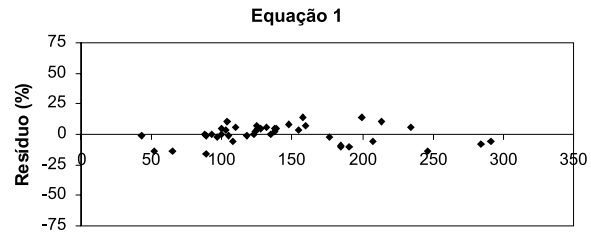

Volume Total com casca ( $\left.\mathrm{m}^{3} / \mathrm{ha}\right)$

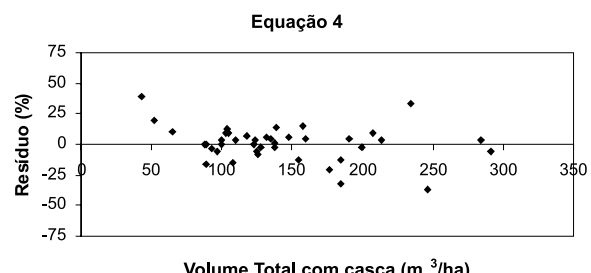

Equação 7

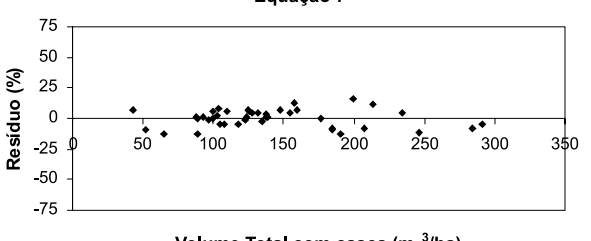

Volume Total com casca $\left(m^{3} / \mathrm{ha}\right)$
$\left(R^{2}=97,59 \%\right)$ e distribuição uniforme dos resíduos.

Ajustando novamente os oito modelos iniciais propostos para o volume do fuste com casca por hectare em função da área basal por hectare e da altura média dos fustes, quando pertinente, obtiveram-se as seguintes estimativas dos parâmetros, bem como as respectivas medidas de precisão (Quadro 9).
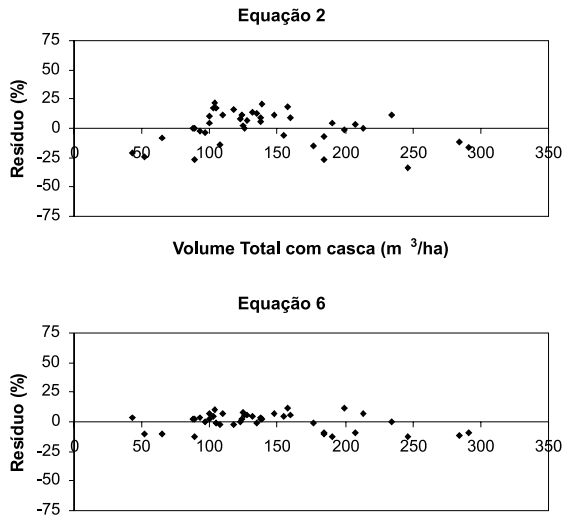

Volume Total com casca $\left(m^{3} / \mathrm{ha}\right)$

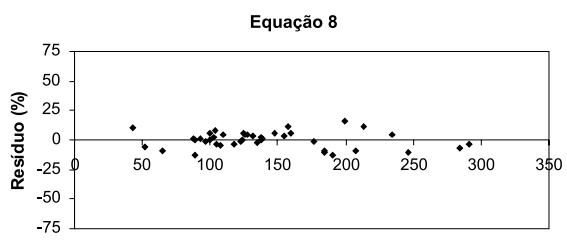

Volume Total com casca $\left(\mathrm{m}^{3} / \mathrm{ha}\right)$

Figura 5 - Distribuição dos resíduos porcentuais para os modelos avaliados. Figure 5 -Distribution of the percentile residues for evaluated models.

R. Árvore, Viçosa-MG, v.29, n.2, p.213-225, 2005 
Equações de volume de povoamento para fragmentos...

Quadro 9 - Equações ajustadas do volume de fuste com casca por hectare $(V f)$ em função da área basal por hectare $(B)$ e da altura média dos fustes $(H f)$ quando pertinente, com os respectivos coeficientes de determinação $\left(R^{2}\right)$ e coeficientes de variação $(C V)$

Table 9 -Adjusted equations for bole volume with bark per hectare ( $V f)$ in relation to basal area per hectare $(B)$ and total average height of the boles $(H f)$ when pertinent, with their respective determination coefficients $\left(R^{2}\right)$ and variation coefficients $(\mathrm{CV})$

\begin{tabular}{|c|c|c|c|}
\hline № & Equações & $R^{2}(\%)$ & $C V(\%)$ \\
\hline 1 & $V f=-0,539780^{n . s}+0,419716^{*} B \cdot H f$ & 97,95 & 5,90 \\
\hline 2 & $V f=-30,683542 *+4,118793 * \operatorname{LnB} \cdot H f$ & 92,16 & 11,54 \\
\hline 3 & $V f=-168,469445 *+79,496438 * \operatorname{Ln} B$ & 74,75 & 20,70 \\
\hline 4 & $\operatorname{LnVf}=2,415674 *+0,071985 * \cdot \operatorname{LnB} \cdot H f$ & 93,95 & 16,52 \\
\hline 5 & $\operatorname{LnVf}=-0,286870^{n \cdot s}+1,490854^{*} \cdot \operatorname{LnB}$ & 86,81 & 21,06 \\
\hline 6 & $\operatorname{LnVf}=-0,987167 *+0,962303 * \cdot \operatorname{Ln} B+1,106652 * \operatorname{LnHf}$ & 99,11 & 5,64 \\
\hline 7 & $V f=-9,037213^{n . s}+O, 078123^{n . s} \cdot B+1,795238^{n . s} . H f+O, 374442^{*} \cdot B . H f$ & 98,12 & 5,65 \\
\hline 8 & $V f=1,263221^{*} \cdot B^{0,219366^{*}} \cdot H f^{0,259838^{*}}$ & 98,93 & 6,16 \\
\hline
\end{tabular}

* Valores dos coeficientes significativos a $5 \%$, pelo teste t.

n.s Valores dos coeficientes não-significativos a $5 \%$, pelo teste $\mathrm{t}$.

Analisando as medidas de precisão das equações no Quadro 9, o comportamento gráfico da distribuição dos resíduos porcentuais na Figura 6 e a significância dos parâmetros, verificou-se novamente que o modelo

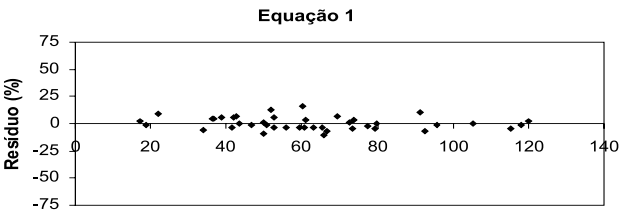

Volume do fuste com casca $\left(m^{3} / h a\right)$

Equação 3

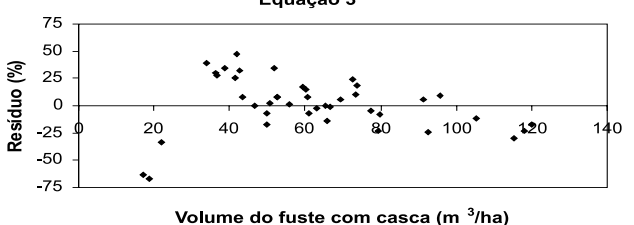

Equação 5

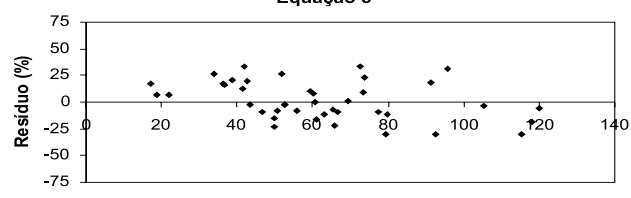

Volume do fuste com casca (m $\left.3 / h^{3}\right)$

Equação 7

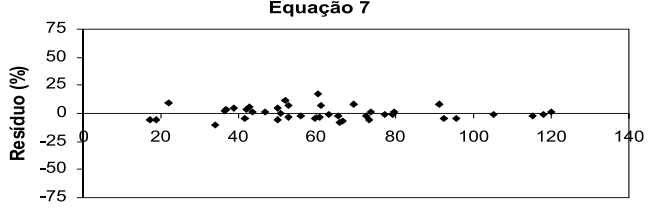

Volume do fuste com casca $\left(m^{3} / h a\right)$
6 foi o melhor para estimar o volume do fuste por hectare. Novamente, verificou-se o problema da multicolinearidade no modelo 7, embora este apresente alto coeficiente de determinação $\left(R^{2}\right)$.

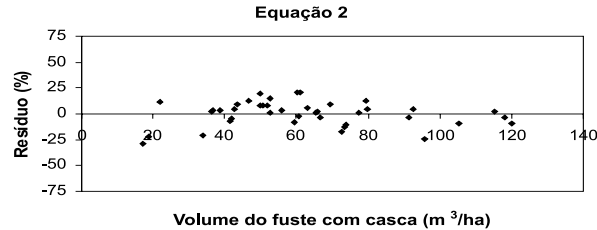

Volume do fuste com casca $\left(m^{3} / \mathrm{ha}\right)$

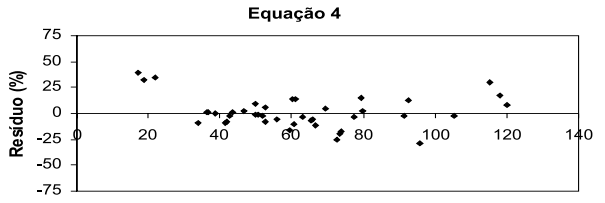

Volume do fuste com casca ( $\left.m^{3} / \mathrm{ha}\right)$

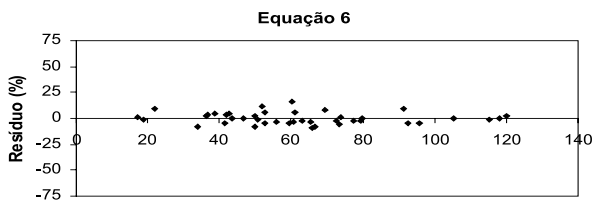

Volume do fuste com casca $\left(m^{3} / h a\right)$

Equação 8

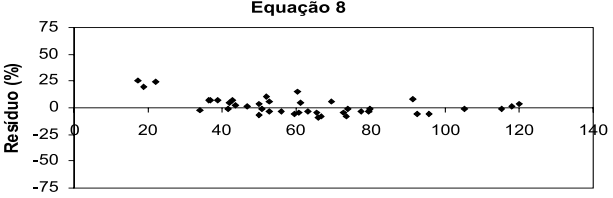

Volume do fuste com casca (m ${ }^{3 / h a)}$

Figura 6 - Distribuição dos resíduos porcentuais para os modelos avaliados. Figure 6 -Distribution of the percentile residues for evaluated models. 


\subsection{Validação das equações ajustadas}

Utilizando dados observados em 14 pontos de amostragem, selecionados aleatoriamente em fragmentos na região de Viçosa e que não foram utilizados nos ajustes dos modelos avaliados, procedeu-se à validação dos modelos selecionados para estimar o volume total com casca por hectare e o volume de fuste com casca por hectare, através da análise gráfica dos resíduos (Figura 7 A, B e C). Através da análise gráfica, verificouse que os modelos selecionados apresentaram leve tendência de subestimação dos volumes, porém com uma discrepância (resíduo) em relação aos valores observados menor que $20 \%$.

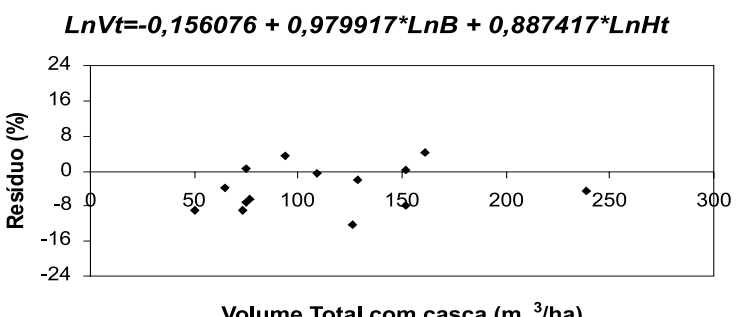

(A)

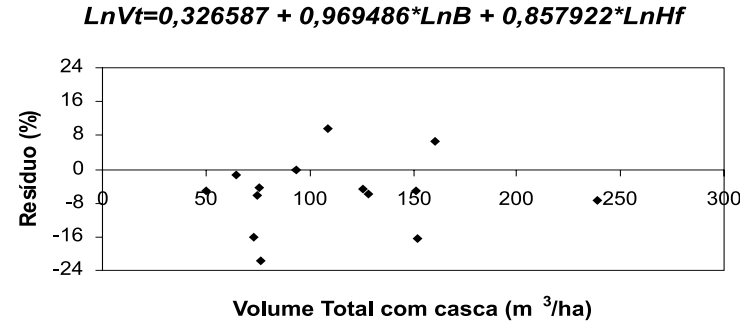

(B)

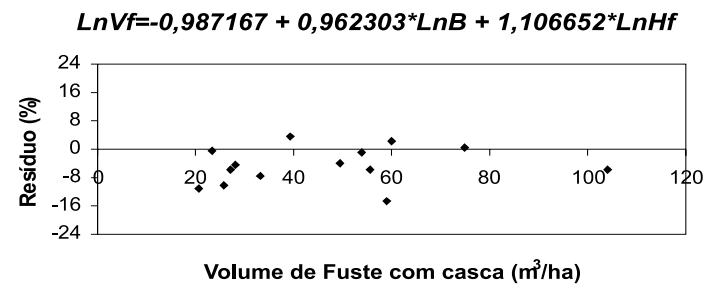

(C)

Figura 7 - Distribuição porcentual dos resíduos em função dos volumes totais com casca por hectare e dos volumes dos fustes por hectare para as equações selecionadas.

Figure 7 - Percentage distribution of residues in function of total volumes per hectare and of bole volumes per hectare for the selected equations.

\section{CONCLUSÕES}

De acordo com os resultados apresentados no presente trabalho, pode-se concluir que:

a) O município de Viçosa possui fragmentos com grande número de espécies (480), pertencentes a 189 gêneros e 62 famílias.

b) Foram contabilizados 289 fragmentos florestais no município de Viçosa, recobrindo $22,77 \%$ da área do município, isto é, $6.832,58$ ha.

c) O modelo cujos volumes por hectare e as variáveis independentes área basal por hectare e altura do povoamento (altura total média e altura média dos fustes) estavam linearizados, foi selecionado como o melhor para estimar o volume total com casca por hectare e o volume de fuste com casca por hectare.

\section{REFERÊNCIAS BIBLIOGRÁFICAS}

\section{ALMEIDA JÜNIOR, J. S. Florística e \\ fitossociologia de fragmentos da floresta estacional semidecidual, Viçosa, Minas Gerais. 1999. 148f. \\ Dissertação (Mestrado em Ciência Florestal) - Universidade Federal de Viçosa, Viçosa, 1999.}

CAMPOS, A. L. A. S. Desenvolvimento de um sistema compatível de crescimento e produção para manejo de Eucalyptus grandis (W. Hill ex. Maiden). 1986. $73 \mathrm{f}$. Dissertação (Mestrado em Ciência Florestal) Universidade Federal de Viçosa, Viçosa, 1986.

CARRON, L.T.; CROMER, D. A. N. Stand volume tables with particular reference to Pinus radiata in the A.C.T. Camberra. Forestry and Timber Bureau, 1956. (Bulletim, 35).

CETEC. Desenvolvimento de equações volumétricas aplicáveis ao manejo sustentado de florestas nativas do estado de Minas Gerais e outras regiões do país. Belo Horizonte: 1995.

CHATURVEDI, M. D. Measurements of Forest crops. London: Oxford University Press, 1926. 142p.

R. Árvore, Viçosa-MG, v.29, n.2, p.213-225, 2005 
COELHO, D. J. S.; SOUZA, A. Modelo de gestão florestal sustentável para a microrregião de Viçosa, Minas Gerais. Revista Árvore, v. 26, n. 2, p. 135-144, 2002.

COLE, D.M. A cubic-foot stand volume equation for Lodgepole pine in Montana and Idaho. Ogden, Utah: United States Department of Agriculture Forest Service Research,. 1971. 8p. (Note, 150).

CORRÊA, G.F. Modelo de evolução e mineralogia da fração argila de solos do planalto de Viçosa - MG. 1984. 187f. Dissertação (Mestrado em Ciência Florestal) Universidade Federal de Viçosa, Viçosa, 1984.

DAVIS, L. S.; JOHNSON, K. M. Forest management. New York: McGraw-Hill Book Company, 1986. 790p.

DEPARTAMENTO NACIONAL DE METEOROLOGIA. Normais climatológicos (1961 - 1990). Brasília: 1991. 84p.

EMPRESA BRASILEIRA DE PESQUISA AGROPECUÁRIA - EMBRAPA. Sistema Brasileiro de Classificação de Solos. Rio de Janeiro: Embrapa Solos, 1999. 412p

FIGUEIREDO FILHO, A. Estudos de modelos matemáticos para estimar o volume por unidade de área em uma floresta tropical úmida na Amazônia brasileira. 1983. 150f. Dissertação (Mestrado em Engenharia Florestal) - Universidade Federal do Paraná, Curitiba, 1983.

GOLFARI, L. Zoneamento ecológico do Estado de Minas Gerais. Belo Horizonte: Centro de pesquisa florestal da Região do Cerrado, 1975. 65p.

HUSH, B.; MILLER, C. I.; BEERS, T. W. Forest mensuration. 2 ed. New York: The Ronald Press Company, 1972. 410 p.

INSTITUTO BRASILEIRO DO MEIO AMBIENTE IBAMA. Flora. Disponível em: <http:// www.ibama.gov.br>. Acesso em: 19 de maio 2002.

INSTITUTO BRASILEIRO DE GEOGRAFIA E ESTATÍSTICA - IBGE. Cidades@. Disponível em: $<$ http://www.ibge.gov.br>. Acesso em: $14 \mathrm{de}$ setembro 2002.
OLIVEIRA, M.L.R. Tabelas de volume de povoamento para fragmentos florestais nativos do município de Viçosa, Minas Gerais. 2003. 80f. Dissertação (Mestrado em Ciência Florestal) - Universidade Federal de Viçosa, Viçosa, 2003.

PEREIRA, R.A. Mapeamento e caracterização de fragmentos de vegetação arbórea e alocação de áreas preferências para sua interligação no município de Viçosa, MG. 1999. 250f. Tese (Doutorado em Ciência Florestal) -Universidade Federal de Viçosa, Viçosa, 1999.

REZENDE, S. B. Estudo de Cronotoposeqüência em Viçosa - MG. 1971. 71f. Dissertação (Mestrado em Solos) Universidade Federal de Viçosa, Viçosa, 1971.

SAUNDERS, D. A.; HOBBS, R.J.; MARGULES, C.R. Biological consequences of ecosystem fragmentation: a review. Conservation Biology, v.5, p18-32,1991

SPURR, S. H. Forest inventory. New York: The Ronald Press, 1952. 476p.

UNG, C. H.; OUELLET, D. Stand volume tables. Application to black spruce of Lebel-surQuevillon. Forestry Chronicle, v.67, n.6, p.712-715, 1991.

VELLOSO, H.P.; RANGEL FILHO, A.L.R.; LIMA, J.C.A. Classificação da vegetação brasileira, adaptada a um sistema universal. Rio de Janeiro: IBGE, 1991. 123p.

VIANA, V.M.; PINHEIRO, L.A.F.V. Conservação da biodiversidade em fragmentos florestais. IPEF, v. 12, n.32, p.25-42, 1998.

VIANA, V.M.; TABANEZ, A. J. A.; MARTINEZ, J.L.A. Restauração e Manejo de Fragmentos Florestais. In: CONGRESSO NACIONAL SOBRE ESSÊNCIAS NATIVAS, 2., 1992, Campos de Jordão. Anais... Campos de Jordão: Instituto Florestal, Secretaria do Meio Ambiente, 1992. v.2, p.400-406.

R. Árvore, Viçosa-MG, v.29, n.2, p.213-225, 2005 